\title{
Attractors for a Class of Kirchhoff Models with $p$-Laplacian and Time Delay
}

\section{Sun-Hye Park}

\begin{abstract}
This paper is concerned with a class of Kirchhoff models with time delay and perturbation of $p$-Laplacian type

$$
u_{t t}(x, t)+\Delta^{2} u(x, t)-\Delta_{p} u(x, t)-a_{0} \Delta u_{t}(x, t)+a_{1} u_{t}(x, t-\tau)+f(u(x, t))=g(x),
$$

where $\Delta_{p} u=\operatorname{div}\left(|\nabla u|^{p-2} \nabla u\right)$ is the usual $p$-Laplacian operator. Many researchers have studied well-posedness and decay rates of energy for these equations without delay effects. But, there are not many studies on attractors for other delayed systems. Thus we establish the existence of global attractors and the finite dimensionality of the attractors by establishing some functionals which are related to the norm of the phase space to our problem.
\end{abstract}

\section{Introduction}

We consider the following Kirchhoff models with time delay and perturbation of $p$-Laplacian type

$$
\begin{gathered}
u_{t t}+\Delta^{2} u-\Delta_{p} u-a_{0} \Delta u_{t}+a_{1} u_{t}(x, t-\tau)+f(u)=g(x) \quad \text { in } \Omega \times \mathbb{R}^{+}, \\
u=\Delta u=0 \quad \text { on } \partial \Omega \times \mathbb{R}^{+}, \\
u(0)=u_{0}, \quad u_{t}(0)=u_{1} \quad \text { on } \Omega, \\
u_{t}(x, t)=j_{0}(x, t) \quad \text { for }(x, t) \in \Omega \times(-\tau, 0),
\end{gathered}
$$

where $\Omega \subset \mathbb{R}^{N}$ is a bounded domain with smooth boundary $\partial \Omega$. $a_{0}>0, a_{1} \in \mathbb{R}, \tau>0$ is time delay, and $g \in L^{2}(\Omega)$.

This model can be regarded as a fourth order viscoelastic plate equation with a lower order perturbation of $p$-Laplacian type and is related to one-dimensional nonlinear equation of elastoplastic microstructure flows given by

$$
u_{t t}+u_{x x x x}-a\left(u_{x}^{2}\right)_{x}=0 .
$$

As a general form of 1 1.5), many authors [8, 14, 16, 17] studied the following equation with appropriate boundary and initial conditions:

$$
u_{t t}+\alpha \Delta^{2} u-\Delta_{p} u-\Delta u_{t}+h\left(u_{t}\right)+f(u)=g(x) .
$$

Received September 11, 2017; Accepted November 11, 2018.

Communicated by Jann-Long Chern.

2010 Mathematics Subject Classification. 35L70, 35B41.

Key words and phrases. attractor, finite dimensionality, time delay, Kirchhoff model, $p$-Laplacian. 
Ma and Soriano [8 proved the global existence and decay of the solutions when $\alpha=$ $h\left(u_{t}\right)=0$ in (1.6). Park et al. [14 improved the results in [8 by generalizing the assumptions on $h$, that is, the function $h$ is assumed to be a discontinuous and nonlinear multi-valued function.

With respect to plate equations with memory of the form

$$
u_{t t}+\Delta^{2} u-\Delta_{p} u+\int_{0}^{t} \mu(t-s) \Delta u^{2}(s) d s-\Delta u_{t}=0
$$

the authors of [1] proved the existence result and established the exponential decay rate when the relaxation function $\mu$ decays at the same rate. The interaction of the memory term with $p$-Laplacian operator was first considered by them. Later, Park [12] obtained general decay rate by weakening the conditions of kernel function $\mu$.

It is well known that the strong damping $-\Delta u_{t}$ plays an important role to obtain global well-posedness and uniqueness of solutions due to the presence of the $p$-Laplacian. Most recently, Jorge silva et al. [6] studied the nonlinear viscoelastic Kirchhoff plate equation of the form

$$
u_{t t}-\Delta u_{t t}+\alpha \Delta^{2} u-\operatorname{div}\left(F\left(|\nabla u|^{2}\right) \nabla u\right)-\int_{0}^{t} \mu(t-s) \Delta^{2} u(s) d s=0,
$$

where $F: \mathbb{R}^{N} \rightarrow \mathbb{R}^{N}$ is a vector field satisfying some conditions, here $|\nabla u|^{p-2}$ is an example of $F(|\nabla u|)$. Dropping the strong damping, they proved the existence and uniqueness of stronger solutions in the presence of the rotational inertia term $-\Delta u_{t t}$, which gives the regularity of solutions and hence enables to control the the $p$-Laplacian term, and showed the memory component is enough to decay the energy of solutions. But, the energy of solutions is stationary when $\mu=0$ in 1.7 . So, it will be needed additional dissipation to investigate energy decay rates or the long time dynamics in terms of attractors.

The aim of this work is to investigate the existence of attractors and finite dimensionality of the attractors for the Kirchhoff equations 1.1 - 1.4 with $p$-Laplacian and time-delay and without the memory.

Since time delays arise in many applications depending not only on the present state but also on some past occurrences and the presence of delay may be a source of instability, partial differential equations with time delay effects have become an active area of research (see [3, 9 11] and references therein). Nicaise and Pignotti [10 investigated the wave equation with time delay

$$
u_{t t}(x, t)-\Delta u(x, t)+a_{0} u_{t}(x, t)+a_{1} u_{t}(x, t-\tau)=0 .
$$

They proved that the energy of the problem decays exponentially under the condition $0<a_{1}<a_{0}$. And then they extended the result to the time varying delay case in 11 . 
For the related works of equations with time delay, we also refer $7,13,16$ and references therein.

It is worth mentioning that there are not much literature on attractors for delayed systems. Furthermore, as far as we are concerned, this is the first work in the literature that takes into account the global attractors for Kirchhoff models with $p$-Laplacian and time delay. To obtain our desired results, we establish some functionals which are related to the norm of the phase space to problem (1.1)-(1.4).

The outline of this paper is as follows. In Section 2, we give some notations and material needed for our work. In Section 3, we prove the existence of attractors for problem (1.1)-(1.4). Finally, in Section 4, we examine the finite dimensionality of the attractors.

\section{Preliminaries}

We denote the inner product in $L^{2}(\Omega)$ by $(\cdot, \cdot)$ and the usual norm of $L^{p}(\Omega)$ by $\|\cdot\|_{p}$. For simplicity, we denote $\|\cdot\|_{2}$ by $\|\cdot\|$. For a Banach space $X,\|\cdot\|_{X}$ denotes the norm of $X$. Let $\lambda$ and $\tilde{\lambda}$ be the best constants satisfying $\lambda\|u\|^{2} \leq\|\Delta u\|^{2}$ for $u \in H^{2}(\Omega) \cap H_{0}^{1}(\Omega)$ and $\tilde{\lambda}\|u\|^{2} \leq\|\nabla u\|^{2}$ for $u \in H_{0}^{1}(\Omega)$, respectively.

We present the precise hypotheses to problem $(1.1)-(1.4)$.

(H1) We assume that $p$ satisfy

$$
2 \leq p \leq \frac{2 N-2}{N-2} \text { if } N \geq 3 \text { and } \quad p \geq 2 \text { if } N=1,2 .
$$

This condition guarantees

$$
H^{2}(\Omega) \cap H_{0}^{1}(\Omega) \hookrightarrow W_{0}^{1,2(p-1)}(\Omega) \hookrightarrow H_{0}^{1}(\Omega) \hookrightarrow L^{2}(\Omega) .
$$

(H2) The forcing term $f: \mathbb{R} \rightarrow \mathbb{R}$ satisfies

$$
\begin{gathered}
|f(u)-f(\widetilde{u})| \leq l\left(1+|u|^{m}+|\widetilde{u}|^{m}\right)|u-\widetilde{u}| \quad \text { for } u, \widetilde{u} \in \mathbb{R}, \\
-l_{0} \leq F(u) \leq f(u) u \quad \text { for } u \in \mathbb{R},
\end{gathered}
$$

here $F(u)=\int_{0}^{u} f(s) d s, l>0, l_{0}>0$, and

$$
0<m \leq \frac{4}{N-4} \text { if } N \geq 5 \text { and } m>0 \text { if } 1 \leq N \leq 4
$$

The condition $(2.3)$ ensures that $H^{2}(\Omega) \hookrightarrow L^{2(m+1)}(\Omega)$.

(H3) $g \in L^{2}(\Omega)$ and $j_{0} \in L^{2}(\Omega \times(-\tau, 0))$.

(H4) The coefficients $a_{0}$ and $a_{1}$ satisfy

$$
0<\left|a_{1}\right|<a_{0} \tilde{\lambda} .
$$




\subsection{Well-posedness}

As in [10, we define the function $z$ as

$$
z(x, \rho, t)=u_{t}(x, t-\rho \tau) \quad \text { for }(x, \rho, t) \in \Omega \times(0,1) \times(0, \infty) .
$$

Then problem (1.1)-(1.4) is equivalent to

$$
\begin{gathered}
u_{t t}+\Delta^{2} u-\Delta_{p} u-a_{0} \Delta u_{t}+a_{1} z(x, 1, t)+f(u)=g(x) \quad \text { on } \Omega \times \mathbb{R}^{+}, \\
\tau z_{t}(x, \rho, t)+z_{\rho}(x, \rho, t)=0 \quad \text { for }(x, \rho, t) \in \Omega \times(0,1) \times(0, \infty), \\
u(x, t)=\Delta u(x, t)=0 \quad \text { for }(x, t) \in \partial \Omega \times \mathbb{R}^{+}, \\
u(x, 0)=u_{0}(x), \quad u_{t}(x, 0)=u_{1}(x) \quad \text { for } x \in \Omega \\
z(x, \rho, 0)=j_{0}(x,-\rho \tau):=z_{0}(x, \rho) \quad \text { for }(x, \rho) \in \Omega \times(0,1) .
\end{gathered}
$$

Let

$$
V_{0}=L^{2}(\Omega), \quad V_{1}=H_{0}^{1}(\Omega), \quad V_{2}=H^{2}(\Omega) \cap H_{0}^{1}(\Omega)
$$

and define the phase space

$$
\mathcal{H}=V_{2} \times V_{0} \times L^{2}(\Omega \times(0,1))
$$

equipped with the norm

$$
\|(u, v, z)\|_{\mathcal{H}}^{2}=\|\Delta u\|^{2}+\|v\|^{2}+\|z\|_{L^{2}(\Omega \times(0,1))}^{2} .
$$

We now state the well-posedness result which can be established by combining the arguments of 4,15 .

Theorem 2.1. Assume that (H1), (H2), (H3) hold. Then we have:

(i) For every $\left(u_{0}, u_{1}, z_{0}\right) \in \mathcal{H}$ and $T>0$, there exists a weak solution $\left(u, u_{t}, z\right)$ of problem 2.4 2.8 in the class

$u \in L^{\infty}\left(0, T ; V_{2}\right), \quad u_{t} \in L^{\infty}\left(0, T ; V_{0}\right) \cap L^{2}\left(0, T ; V_{1}\right), \quad z \in L^{\infty}\left(0, T ; L^{2}(\Omega \times(0,1))\right)$

satisfying $\left(u, u_{t}, z\right) \in C([0, T] ; \mathcal{H})$. Moreover, the solution is unique and depends continuously on the initial data $\left(u_{0}, u_{1}, z_{0}\right) \in \mathcal{H}$ and $g \in L^{2}(\Omega)$.

(ii) Let $\left(u, u_{t}, z\right)$ and $\left(\widetilde{u}, \widetilde{u}_{t}, \widetilde{z}\right)$ be two weak solutions of problem 2.4 -2.8 corresponding to initial data $\left(u_{0}, u_{1}, z_{0}\right)$ and $\left(\widetilde{u}_{0}, \widetilde{u}_{1}, \widetilde{z}_{0}\right)$, respectively. Then one gets

$$
\left.\left\|\left(u, u_{t}, z\right)-\left(\widetilde{u}, \widetilde{u}_{t}, \widetilde{z}\right)\right\|_{\mathcal{H}} \leq e^{c t} \|\left(u_{0}, u_{1}, z_{0}\right)-\left(\widetilde{u}_{0}, \widetilde{u}_{1}, \widetilde{z}_{0}\right)\right) \|_{\mathcal{H}} \quad \text { for some } c>0 \text {. }
$$


2.2. A short overview on infinite-dimensional dynamical systems

To study the existence of attractors for problem (2.4)-2.8 and the finite dimensionality of the attractors, we present some basic concepts and abstract results on dynamical systems given by the Chueshov and Lasiecka's book [2].

Let $\mathcal{F}$ be a Banach space and $B$ be a bounded subset of $\mathcal{F}$. We call a function $\phi(\cdot, \cdot)$ which defined on $\mathcal{F} \times \mathcal{F}$ is a contractive function on $B \times B$ if for any sequence $\left\{x_{n}\right\}_{n=1}^{\infty} \subset B$, there is a subsequence $\left\{x_{n_{k}}\right\}_{k=1}^{\infty} \subset\left\{x_{n}\right\}_{n=1}^{\infty}$ such that

$$
\liminf _{k \rightarrow \infty} \liminf _{l \rightarrow \infty} \phi\left(x_{n_{k}}, x_{n_{l}}\right)=0
$$

Theorem 2.2. 2, Theorem 7.1.11] Let $\{S(t)\}_{t \geq 0}$ be a semigroup on a Banach space $\left(\mathcal{F},\|\cdot\|_{\mathcal{F}}\right)$ and have a bounded absorbing set $B_{0}$. Assume that for any $\epsilon>0$ there exist $T=T\left(B_{0}, \epsilon\right)$ and a contractive function $\phi_{T}(\cdot, \cdot)$ on $B_{0} \times B_{0}$ such that

$$
\|S(T) x-S(T) y\|_{\mathcal{F}} \leq \epsilon+\phi_{T}(x, y) \quad \text { for all } x, y \in B_{0},
$$

where $\phi_{T}$ depends on $T$. Then $S(t)$ is asymptotically smooth in $\mathcal{F}$.

Theorem 2.3. [2, Theorem 7.2.4] A dissipative dynamical system $(S(t), \mathcal{F})$ has a compact global attractor if and only if it is asymptotically smooth.

Let $X, Y, Z$ be three reflexive Banach spaces with $X$ compactly embedded in $Y$, $\mathcal{F}=X \times Y \times Z$, and $(S(t), \mathcal{F})$ a dynamical system given by an evolution operator

$$
S(t) x=\left(u(t), u_{t}(t), z(t)\right) \text { for } x=\left(u_{0}, u_{1}, z_{0}\right) \in \mathcal{F},
$$

where the functions $u$ has regularity

$$
u \in C\left(\mathbb{R}^{+} ; X\right) \cap C\left(\mathbb{R}^{+} ; Y\right), \quad z \in C\left(\mathbb{R}^{+} ; Z\right) .
$$

We call the dynamical system $(S(t), \mathcal{F})$ is quasi-stable on $B \subset \mathcal{F}$ if there exists a compact seminorm $n_{X}$ on $X$ and nonnegative scalar function $a(t)$ and $c(t)$, locally bounded in $[0, \infty)$, and $b(t) \in L^{1}\left(\mathbb{R}^{+}\right)$with $\lim _{t \rightarrow \infty} b(t)=0$ such that

$$
\|S(t) x-S(t) y\|_{\mathcal{F}}^{2} \leq a(t)\|x-y\|_{\mathcal{F}}^{2}
$$

and

$$
\|S(t) x-S(t) y\|_{\mathcal{F}}^{2} \leq b(t)\|x-y\|_{\mathcal{F}}^{2}+c(t) \sup _{0<s<t}\left[n_{X}(u(s)-\widetilde{u}(s))\right]^{2},
$$

where $S(t) x=\left(u(t), u_{t}(t), z(t)\right), S(t) y=\left(\widetilde{u}(t), \widetilde{u}_{t}(t), \widetilde{z}(t)\right)$ and $x, y \in B$.

Theorem 2.4. [2, Theorem 7.9.6] Let $(S(t), \mathcal{F})$ be given by 2.9) and satisfy 2.10). If $(S(t), \mathcal{F})$ has a compact global attractor $\mathcal{A}$ and is quasi-stable on $\mathcal{A}$, then the attractor $\mathcal{A}$ has finite fractional dimension. 


\section{Existence of attractors}

In this section we prove the existence of global attractors for problem $2.4-(2.8)$ by applying Theorem 2.3. For this, let us define a map $S(t): \mathcal{H} \rightarrow \mathcal{H}$ by

$$
S(t)\left(u_{0}, u_{1}, z_{0}\right)=\left(u(t), u_{t}(t), z(t)\right)
$$

where $\left(u(t), u_{t}(t), z(t)\right)$ is the unique weak solution of system (2.4)-2.8 corresponding to initial data $\left(u_{0}, u_{1}, z_{0}\right)$. Then, by Theorem 2.1. $\{S(t)\}_{t \geq 0}$ is a $C^{0}$-semigroup on $\mathcal{H}$.

To obtain our desired result, we need to show that the dynamical system given in (3.1) is dissipative and asymptotically smooth. Inspired by 11, let us define the energy of problem (2.4)-(2.8) by

$$
\begin{aligned}
E(t)= & \frac{1}{2}\left\|u_{t}(t)\right\|^{2}+\frac{1}{2}\|\Delta u(t)\|^{2}+\frac{1}{p}\|\nabla u(t)\|_{p}^{p}+\int_{\Omega} F(u(t)) d x \\
& -(g, u(t))+\frac{\xi}{2} \int_{t-\tau}^{t} e^{\theta(s-t)}\left\|u_{t}(s)\right\|^{2} d s,
\end{aligned}
$$

where

$$
\left|a_{1}\right|<\xi<2 a_{0} \widetilde{\lambda}-\left|a_{1}\right| \text { and } 0<\theta<\frac{1}{\tau} \ln \frac{\xi}{\left|a_{1}\right|}
$$

The lemma below states the relation between the norm of phase space and the energy, which plays an important role in the process of our work.

Lemma 3.1. There exists a positive constant $c_{0}$ such that

$$
\left\|\left(u, u_{t}, z\right)\right\|_{\mathcal{H}}^{2} \leq c_{0}\left(E(t)+l_{0}|\Omega|+\frac{\|g\|^{2}}{\lambda}\right) .
$$

Proof. Integration by substitution $s=t-\rho \tau$ implies that

$$
\begin{aligned}
\frac{\xi}{2} \int_{t-\tau}^{t} e^{\theta(s-t)}\left\|u_{t}(s)\right\|^{2} d s & =-\frac{\xi \tau}{2} \int_{1}^{0} \int_{\Omega} e^{-\theta \rho \tau} u_{t}^{2}(x, t-\rho \tau) d x d \rho \\
& =\frac{\xi \tau}{2} \int_{0}^{1} \int_{\Omega} e^{-\theta \rho \tau} z^{2}(x, \rho, t) d x d \rho .
\end{aligned}
$$

Young's inequality and $(2.2)$ give

$$
\int_{\Omega} F(u) d x-(g, u) \geq-l_{0}|\Omega|-\frac{1}{4}\|\Delta u\|^{2}-\frac{\|g\|^{2}}{\lambda} .
$$

Substituting (3.5) and 3.6 into (3.2), one sees that

$$
E(t) \geq \frac{1}{2}\left\|u_{t}\right\|^{2}+\frac{1}{4}\|\Delta u\|^{2}+\frac{1}{p}\|\nabla u\|_{p}^{p}-l_{0}|\Omega|-\frac{\|g\|^{2}}{\lambda}+\frac{\xi \tau}{2} \int_{0}^{1} \int_{\Omega} e^{-\theta \tau \rho} z^{2}(x, \rho, t) d x d \rho
$$




$$
\begin{aligned}
& \geq \frac{1}{2}\left\|u_{t}\right\|^{2}+\frac{1}{4}\|\Delta u\|^{2}+\frac{1}{p}\|\nabla u\|_{p}^{p}-l_{0}|\Omega|-\frac{\|g\|^{2}}{\lambda}+\frac{\xi \tau e^{-\theta \tau}}{2} \int_{0}^{1} \int_{\Omega} z^{2}(x, \rho, t) d x d \rho \\
& \geq \frac{1}{c_{0}}\left(\left\|u_{t}\right\|^{2}+\|\Delta u\|^{2}+\|z\|_{L^{2}(\Omega \times(0,1))}^{2}\right)-l_{0}|\Omega|-\frac{\|g\|^{2}}{\lambda} \\
& =\frac{1}{c_{0}}\left\|\left(u, u_{t}, z\right)\right\|_{\mathcal{H}}-l_{0}|\Omega|-\frac{\|g\|^{2}}{\lambda},
\end{aligned}
$$

where $1 / c_{0}:=\min \left\{1 / 4, \xi \tau e^{-\theta \tau} / 2\right\}$.

Lemma 3.2. Assume that $(\mathrm{H} 1)-(\mathrm{H} 4)$ hold. Then there exists positive constants $c_{1}$ and $c_{2}$ satisfying

$$
E^{\prime}(t) \leq-c_{1}\left\|\nabla u_{t}(t)\right\|^{2}-c_{2}\|z(1, t)\|^{2}-\frac{\theta \xi}{2} \int_{t-\tau}^{t} e^{\theta(s-t)}\left\|u_{t}(s)\right\|^{2} d s
$$

Proof. Multiplying (2.4) by $u_{t}$ integrating over $x \in \Omega$, we get

$$
\begin{aligned}
& \frac{d}{d t}\left(\frac{1}{2}\left\|u_{t}(t)\right\|^{2}+\frac{1}{2}\|\Delta u(t)\|^{2}+\frac{1}{p}\|\nabla u(t)\|_{p}^{p}+\int_{\Omega} F(u(t)) d x-(g, u(t))\right) \\
= & -a_{0}\left\|\nabla u_{t}(t)\right\|^{2}-a_{1}\left(z(1, t), u_{t}(t)\right) .
\end{aligned}
$$

From this, 3.2, and the following estimate

$$
\frac{d}{d t} \int_{t-\tau}^{t} e^{\theta(s-t)}\left\|u_{t}(s)\right\|^{2} d s=-\theta \int_{t-\tau}^{t} e^{\theta(s-t)}\left\|u_{t}(s)\right\|^{2} d s+\left\|u_{t}(t)\right\|^{2}-e^{-\theta \tau}\left\|u_{t}(t-\tau)\right\|^{2}
$$

we see that

$$
\begin{aligned}
E^{\prime}(t)= & -a_{0}\left\|\nabla u_{t}(t)\right\|^{2}-a_{1}\left(z(1, t), u_{t}(t)\right)-\frac{\theta \xi}{2} \int_{t-\tau}^{t} e^{\theta(s-t)}\left\|u_{t}(s)\right\|^{2} d s \\
& +\frac{\xi}{2}\left\|u_{t}(t)\right\|^{2}-\frac{\xi e^{-\theta \tau}}{2}\left\|u_{t}(t-\tau)\right\|^{2} .
\end{aligned}
$$

This and Young's inequality give

$$
\begin{aligned}
E^{\prime}(t) \leq & -\left(a_{0}-\frac{\left|a_{1}\right|}{2 \widetilde{\lambda}}-\frac{\xi}{2 \widetilde{\lambda}}\right)\left\|\nabla u_{t}(t)\right\|^{2}-\left(\frac{\xi e^{-\theta \tau}}{2}-\frac{\left|a_{1}\right|}{2}\right)\|z(1, t)\|^{2} \\
& -\frac{\theta \xi}{2} \int_{t-\tau}^{t} e^{\theta(s-t)}\left\|u_{t}(s)\right\|^{2} d s
\end{aligned}
$$

Letting $c_{1}:=a_{0}-\left|a_{1}\right| /(2 \widetilde{\lambda})-\xi /(2 \widetilde{\lambda})$ and $c_{2}:=\xi e^{-\theta \tau} / 2-\left|a_{1}\right| / 2$, which are positive owing to 3.3 , we complete the proof.

Now, define the perturbed functional by

$$
L(t)=E(t)+\epsilon \Phi(t)
$$


where $\Phi(t)=\left(u_{t}(t), u(t)\right)$, then one sees from Young's inequality and (3.4) that

$$
|\Phi(t)| \leq \frac{1}{2}\left\|u_{t}(t)\right\|^{2}+\frac{1}{2 \lambda}\|\Delta u(t)\|^{2} \leq c_{0} \max \left\{\frac{1}{2}, \frac{1}{2 \lambda}\right\}\left(E(t)+l_{0}|\Omega|+\frac{\|g\|^{2}}{\lambda}\right) .
$$

So, it holds that

$$
|L(t)-E(t)| \leq \epsilon c\left(E(t)+l_{0}|\Omega|+\frac{\|g\|^{2}}{\lambda}\right),
$$

here and in the sequel $c$ denotes a generic positive constant different from line to line or even in the same line. Choosing $\epsilon>0$ small, we deduce that

$$
\alpha_{1} E(t)-c_{3}\left(l_{0}|\Omega|+\frac{\|g\|^{2}}{\lambda}\right) \leq L(t) \leq \alpha_{2} E(t)+c_{3}\left(l_{0}|\Omega|+\frac{\|g\|^{2}}{\lambda}\right) \quad \text { for } t \geq 0
$$

where $\alpha_{1}=1-\epsilon c, \alpha_{2}=1+\epsilon c$, and $c_{3}=\epsilon c$.

Lemma 3.3. Assume the conditions (H1)-(H4) hold. Then, the semigroup $\{S(t)\}_{t \geq 0}$ defined by 3.1 has a bounded absorbing set in $\mathcal{H}$.

Proof. We have from (2.4) that

$$
\begin{aligned}
\Phi^{\prime}(t)= & \left\|u_{t}(t)\right\|^{2}-\|\Delta u(t)\|^{2}-\|\nabla u(t)\|_{p}^{p}+a_{0}\left(u_{t}(t), \Delta u(t)\right) \\
& -a_{1}(u(t), z(1, t))-(f(u(t)), u(t))+(g, u(t)) .
\end{aligned}
$$

Young's inequality gives that

$$
\begin{gathered}
a_{0}\left(u_{t}(t), \Delta u(t)\right) \leq a_{0}^{2}\left\|u_{t}(t)\right\|^{2}+\frac{1}{4}\|\Delta u(t)\|^{2}, \\
-a_{1}(u(t), z(1, t)) \leq \frac{1}{4}\|\Delta u(t)\|^{2}+\frac{a_{1}^{2}}{\lambda}\|z(1, t)\|^{2} .
\end{gathered}
$$

Substituting these into $(3.9)$ and applying $(2.2)$, we find

$$
\Phi^{\prime}(t) \leq c_{4}\left\|u_{t}(t)\right\|^{2}-\frac{1}{2}\|\Delta u(t)\|^{2}-\|\nabla u(t)\|_{p}^{p}+c_{5}\|z(1, t)\|^{2}-\int_{\Omega} F(u) d x+(g, u(t))
$$

where $c_{4}=1+a_{0}^{2}$ and $c_{5}=a_{1}^{2} / \lambda$. Thanks to Lemma 3.2 and 3.10 , we see that

$$
\begin{aligned}
L^{\prime}(t) \leq & -c_{1}\left\|\nabla u_{t}(t)\right\|^{2}-c_{2}\|z(1, t)\|^{2}-\frac{\theta \xi}{2} \int_{t-\tau}^{t} e^{\theta(s-t)}\left\|u_{t}(s)\right\|^{2} d s+\epsilon c_{4}\left\|u_{t}(t)\right\|^{2} \\
& -\frac{\epsilon}{2}\|\Delta u(t)\|^{2}-\epsilon\|\nabla u(t)\|_{p}^{p}+\epsilon c_{5}\|z(1, t)\|^{2}-\epsilon \int_{\Omega} F(u) d x+\epsilon(g, u(t)) \\
\leq & -\left(c_{1}-\frac{2 \epsilon c_{4}}{\widetilde{\lambda}}\right)\left\|\nabla u_{t}(t)\right\|^{2}-\epsilon c_{4}\left\|u_{t}(t)\right\|^{2}-\frac{\epsilon}{2}\|\Delta u(t)\|^{2}-\epsilon\|\nabla u(t)\|_{p}^{p} \\
& -\epsilon \int_{\Omega} F(u) d x+\epsilon(g, u(t))-\frac{\theta \xi}{2} \int_{t-\tau}^{t} e^{\theta(s-t)}\left\|u_{t}(s)\right\|^{2} d s-\left(c_{2}-\epsilon c_{5}\right)\|z(1, t)\|^{2} .
\end{aligned}
$$


Choosing $\epsilon>0$ small enough such that $c_{1}-2 \epsilon c_{4} / \widetilde{\lambda}>0, c_{2}-\epsilon c_{5}>0$, we deduce that

$$
L^{\prime}(t) \leq-\alpha_{3} E(t) \quad \text { for some } \alpha_{3}>0
$$

From this, (3.8), and (3.4) we infer that

$$
\left\|\left(u(t), u_{t}(t), z\right)\right\|_{\mathcal{H}}^{2} \leq \frac{c_{0} \alpha_{2}}{\alpha_{1}} E(0) e^{-\alpha_{3} t / \alpha_{2}}+c_{0}\left(\frac{2 c_{3}}{\alpha_{1}}+1\right)\left(l_{0}|\Omega|+\frac{\|g\|^{2}}{\lambda}\right) .
$$

This shows that any closed ball $B_{0}=\bar{B}(0, R)$ with $R>\sqrt{c_{0}\left(2 c_{3} / \alpha_{1}+1\right)\left(l_{0}|\Omega|+\|g\|^{2} / \lambda\right)}$ is a bounded absorbing set of $(S(t), \mathcal{H})$.

Lemma 3.4. Assume the conditions (H1)-(H4) hold. Let $B_{0}$ be a bounded absorbing set obtained in Lemma 3.3, $S(t) y_{0}=\left(u, u_{t}, z\right)$ and $S(t) \widetilde{y}_{0}=\left(\widetilde{u}, \widetilde{u}_{t}, \widetilde{z}\right)$ be two weak solutions of problem (2.4) -2.8) corresponding to initial data $y_{0}=\left(u_{0}, u_{1}, z_{0}\right) \in B_{0}$ and $\widetilde{y_{0}}=$ $\left(\widetilde{u}_{0}, \widetilde{u}_{1}, \widetilde{z}_{0}\right) \in B_{0}$, respectively. Then,

$$
\begin{aligned}
& \left\|S(t) y_{0}-S(t) \widetilde{y_{0}}\right\|_{\mathcal{H}}^{2} \\
\leq & c e^{-\omega t}\left\|y_{0}-\widetilde{y_{0}}\right\|_{\mathcal{H}}^{2} \\
& +C\left(B_{0}\right) \int_{0}^{t} e^{-\omega(t-s)}\left(\|\nabla u(s)-\nabla \widetilde{u}(s)\|_{2(p-1)}^{2}+\|u(s)-\widetilde{u}(s)\|_{2(m+1)}^{2}\right) d s,
\end{aligned}
$$

where $c>0, \omega>0$, and $C\left(B_{0}\right)$ is a constant depending on the size of $B_{0}$.

Proof. Let $w(t)=u(t)-\widetilde{u}(t), q(x, \rho, t)=z(x, \rho, t)-\widetilde{z}(x, \rho, t)$. Then from (2.4)-(2.8), $w$ and $q$ satisfy

$$
\begin{aligned}
& w_{t t}+\Delta^{2} w-\left(\Delta_{p} u-\Delta_{p} \widetilde{u}\right)-a_{0} \Delta w_{t}+a_{1} q(x, 1, t)+f(u)-f(\widetilde{u})=0 \quad \text { on } \Omega \times \mathbb{R}^{+}, \\
& \tau q_{t}(x, \rho, t)+q(x, \rho, t)=0 \quad \text { for }(x, \rho, t) \in \Omega \times(0,1) \times(0, \infty), \\
& w=\Delta w=0 \quad \text { on } \partial \Omega \times \mathbb{R}^{+}, \\
& w(0)=u_{0}-\widetilde{u}_{0}, \quad w_{t}(0)=u_{1}-\widetilde{u}_{1} \quad \text { on } \Omega, \\
& q(x, \rho, 0)=z_{0}(x, \rho)-\widetilde{z}_{0}(x, \rho):=q_{0} \quad \text { for }(x, \rho) \in \Omega \times(0,1) .
\end{aligned}
$$

The similar calculation to that of $(3.7)$ yields

$$
\begin{aligned}
E_{w}^{\prime}(t) \leq & -\left(a_{0}-\frac{\left|a_{1}\right|}{2 \widetilde{\lambda}}-\frac{\xi}{2 \widetilde{\lambda}}\right)\left\|\nabla w_{t}(t)\right\|^{2}-\left(\frac{\xi e^{-\theta \tau}}{2}-\frac{\left|a_{1}\right|}{2}\right)\|q(1, t)\|^{2} \\
& -\frac{\theta \xi}{2} \int_{t-\tau}^{t} e^{\theta(s-t)}\left\|w_{t}(s)\right\|^{2} d s-\left(\Delta_{p} u(t)-\Delta_{p} \widetilde{u}(t), w_{t}(t)\right) \\
& -\left(f(u(t))-f(\widetilde{u}(t)), w_{t}(t)\right)
\end{aligned}
$$

where

$$
E_{w}(t)=\frac{1}{2}\left\|w_{t}(t)\right\|^{2}+\frac{1}{2}\|\Delta w(t)\|^{2}+\frac{\xi}{2} \int_{t-\tau}^{t} e^{\theta(s-t)}\left\|w_{t}(s)\right\|^{2} d s
$$


here $\theta$ and $\xi$ are as given in (3.3). In what follows, we shall estimate the last two terms on the right had side of 3.13 . From the Hölder inequality with $\frac{p-2}{2(p-1)}+\frac{1}{2(p-1)}+\frac{1}{2}=1$, we see that

$$
\begin{aligned}
-\left(\Delta_{p} u-\Delta_{p} \widetilde{u}, w_{t}\right) & =\int_{\Omega}\left(|\nabla u|^{p-2} \nabla u-|\nabla \widetilde{u}|^{p-2} \nabla \widetilde{u}\right) \nabla w_{t} d x \\
& \leq c \int_{\Omega}\left(|\nabla u|^{p-2}+|\nabla \widetilde{u}|^{p-2}\right)\left|\nabla w \| \nabla w_{t}\right| d x \\
& \leq c\left(\|\nabla u\|_{2(p-1)}^{p-2}+\|\nabla \widetilde{u}\|_{2(p-1)}^{p-2}\right)\|\nabla w\|_{2(p-1)}\left\|\nabla w_{t}\right\| \\
& \leq \frac{C\left(B_{0}\right)}{2 \eta}\|\nabla w\|_{2(p-1)}^{2}+\frac{\eta}{2}\left\|\nabla w_{t}\right\|^{2} .
\end{aligned}
$$

Using (2.1) and $\frac{m}{2(m+1)}+\frac{1}{2(m+1)}+\frac{1}{2}=1$, we also obtain

$$
\begin{aligned}
-\left(f(u)-f(\widetilde{u}), w_{t}\right) & \leq l c\left(|\Omega|^{\frac{m}{2(m+1)}}+\|u\|_{2(m+1)}^{m}+\|\widetilde{u}\|_{2(m+1)}^{m}\right)\|w\|_{2(m+1)}\left\|w_{t}\right\| \\
& \leq C\left(B_{0}\right)\|w\|_{2(m+1)}\left\|\nabla w_{t}\right\| \\
& \leq \frac{C\left(B_{0}\right)}{2 \eta}\|w\|_{2(m+1)}^{2}+\frac{\eta}{2}\left\|\nabla w_{t}\right\|^{2} .
\end{aligned}
$$

Applying these to 3.13 , we observe

$$
\begin{aligned}
E_{w}^{\prime}(t) \leq & -\left(a_{0}-\frac{\left|a_{1}\right|}{2 \widetilde{\lambda}}-\frac{\xi}{2 \widetilde{\lambda}}-\eta\right)\left\|\nabla w_{t}(t)\right\|^{2}-\left(\frac{\xi e^{-\theta \tau}}{2}-\frac{\left|a_{1}\right|}{2}\right)\|q(1, t)\|^{2} \\
& -\frac{\theta \xi}{2} \int_{t-\tau}^{t} e^{\theta(s-t)}\left\|w_{t}(s)\right\|^{2} d s+\frac{C\left(B_{0}\right)}{2 \eta}\|\nabla w(t)\|_{2(p-1)}^{2} \\
& +\frac{C\left(B_{0}\right)}{2 \eta}\|w(t)\|_{2(m+1)}^{2} .
\end{aligned}
$$

On the other hand, it can be observed that $E_{w}$ is equivalent to $\left\|\left(w, w_{t}, q\right)\right\|_{\mathcal{H}}$. Indeed, integration by substitution $s=t-\rho \tau$ gives

$$
\begin{aligned}
E_{w}(t) & =\frac{1}{2}\left\|w_{t}(t)\right\|^{2}+\frac{1}{2}\|\Delta w(t)\|^{2}+\frac{\xi \tau}{2} \int_{0}^{1} e^{-\theta \rho \tau}\left\|w_{t}(t-\rho \tau)\right\|^{2} d \rho \\
& \geq \frac{1}{2}\left\|w_{t}(t)\right\|^{2}+\frac{1}{2}\|\Delta w(t)\|^{2}+\frac{\xi \tau e^{-\theta \tau}}{2} \int_{0}^{1} \int_{\Omega} q^{2}(x, \rho, t) d x d \rho \\
& \geq \min \left\{\frac{1}{2}, \frac{\xi \tau e^{-\theta \tau}}{2}\right\}\left\|\left(w, w_{t}, q\right)\right\|_{\mathcal{H}}
\end{aligned}
$$

and it holds that

$$
\begin{aligned}
E_{w}(t) & =\frac{1}{2}\left\|w_{t}(t)\right\|^{2}+\frac{1}{2}\|\Delta w(t)\|^{2}+\frac{\xi \tau}{2} \int_{0}^{1} e^{-\theta \rho \tau}\left\|w_{t}(t-\rho \tau)\right\|^{2} d \rho \\
& \leq \frac{1}{2}\left\|w_{t}(t)\right\|^{2}+\frac{1}{2}\|\Delta w(t)\|^{2}+\frac{\xi \tau}{2} \int_{0}^{1} \int_{\Omega} q^{2}(x, \rho, t) d x d \rho \\
& =\max \left\{\frac{1}{2}, \frac{\xi \tau}{2}\right\}\left\|\left(w, w_{t}, q\right)\right\|_{\mathcal{H}} .
\end{aligned}
$$


Next, let us define

$$
L_{w}(t)=E_{w}(t)+\varepsilon \phi(t),
$$

where $\phi(t)=\left(w_{t}(t), w(t)\right)$. It can be easily shown that for appropriately small $\varepsilon>0$ there exist positive constants $\alpha_{6}$ and $\alpha_{7}$ satisfying

$$
\alpha_{6} E_{w}(t) \leq L_{w}(t) \leq \alpha_{7} E_{w}(t)
$$

From 3.12 , it follows

$$
\begin{aligned}
\phi^{\prime}(t)= & \left\|w_{t}(t)\right\|^{2}-\|\Delta w(t)\|^{2}-\left(\Delta_{p} u(t)-\Delta_{p} \widetilde{u}(t), w(t)\right)+a_{0}\left(w_{t}(t), \Delta w(t)\right) \\
& -a_{1}(q(1, t), w(t))-(f(u(t))-f(\widetilde{u}(t)), w(t)) .
\end{aligned}
$$

By Young's inequality, we know that

$$
a_{0}\left(w_{t}, \Delta w\right) \leq \frac{\eta}{2}\|\Delta w\|^{2}+\frac{a_{0}^{2}}{2 \eta}\left\|w_{t}\right\|^{2} \quad \text { and } \quad-a_{1}(q(1, t), w) \leq \frac{\eta}{2}\|\Delta w\|^{2}+\frac{a_{1}^{2}}{2 \eta \lambda}\|q(1, t)\|^{2} .
$$

By the same arguments of (3.14) and (3.15), we also see that

$$
-\left(\Delta_{p} u-\Delta_{p} \widetilde{u}, w\right) \leq C\left(B_{0}\right)\|\nabla w\|_{2(p-1)}\|\nabla w\| \leq C\left(B_{0}\right)\|\nabla w\|_{2(p-1)}^{2},
$$

where we used the embedding $W_{0}^{1,2(p-1)}(\Omega) \hookrightarrow H_{0}^{1}(\Omega)$, and

$$
-(f(u)-f(\widetilde{u}), w) \leq C\left(B_{0}\right)\|w\|_{2(m+1)}\|w\| \leq \frac{C\left(B_{0}\right)}{2 \eta \lambda}\|w\|_{2(m+1)}^{2}+\frac{\eta}{2}\|\Delta w\|^{2} .
$$

Substituting these into (3.18), we deduce

$$
\begin{aligned}
\phi^{\prime}(t) \leq & \left(1+\frac{a_{0}^{2}}{2 \eta}\right)\left\|w_{t}(t)\right\|^{2}-\left(1-\frac{3 \eta}{2}\right)\|\Delta w(t)\|^{2} \\
& +\frac{a_{1}^{2}}{2 \eta \lambda}\|q(1, t)\|^{2}+C\left(B_{0}\right)\|\nabla w(t)\|_{2(p-1)}^{2}+\frac{C\left(B_{0}\right)}{2 \eta \lambda}\|w(t)\|_{2(m+1)}^{2} .
\end{aligned}
$$

Combining (3.16) with (3.19), we have

$$
\begin{aligned}
L_{w}^{\prime}(t) \leq & -\left\{a_{0}-\frac{\left|a_{1}\right|}{2 \widetilde{\lambda}}-\frac{\xi}{2 \widetilde{\lambda}}-\eta-2 \varepsilon\left(1+\frac{a_{0}^{2}}{2 \eta}\right)\right\}\left\|\nabla w_{t}(t)\right\|^{2}-\varepsilon\left(1+\frac{a_{0}^{2}}{2 \eta}\right)\left\|w_{t}(t)\right\|^{2} \\
& -\varepsilon\left(1-\frac{3 \eta}{2}\right)\|\Delta w(t)\|^{2}-\frac{\theta \xi}{2} \int_{t-\tau}^{t} e^{\theta(s-t)}\left\|w_{t}(s)\right\|^{2} d s \\
& -\left(\frac{\xi e^{-\theta \tau}}{2}-\frac{\left|a_{1}\right|}{2}-\varepsilon \frac{a_{1}^{2}}{2 \eta \lambda}\right)\|q(1, t)\|^{2}+\left(\frac{C\left(B_{0}\right)}{2 \eta}+\varepsilon C\left(B_{0}\right)\right)\|\nabla w(t)\|_{2(p-1)}^{2} \\
& +\left(\frac{C\left(B_{0}\right)}{2 \eta}+\frac{\varepsilon C\left(B_{0}\right)}{2 \eta \lambda}\right)\|w(t)\|_{2(m+1)}^{2} .
\end{aligned}
$$

Choosing $\eta>0$ and $\varepsilon>0$ small enough, we infer

$$
L_{w}^{\prime}(t) \leq-c E_{w}(t)+C\left(B_{0}\right)\left(\|\nabla w(t)\|_{2(p-1)}^{2}+\|w(t)\|_{2(m+1)}^{2}\right) .
$$


This and (3.17) yield that

$$
L_{w}^{\prime}(t) \leq-\omega L_{w}(t)+C\left(B_{0}\right)\left(\|\nabla w(t)\|_{2(p-1)}^{2}+\|w(t)\|_{2(m+1)}^{2}\right) \quad \text { for some } \omega>0 .
$$

Thus, we have from this and (3.17) that

$$
E_{w}(t) \leq c e^{-\omega t} E_{w}(0)+C\left(B_{0}\right) \int_{0}^{t} e^{-\omega(t-s)}\left(\|\nabla w(s)\|_{2(p-1)}^{2}+\|w(s)\|_{2(m+1)}^{2}\right) d s .
$$

Owing to $E_{w} \sim\left\|\left(w, w_{t}, q\right)\right\|_{\mathcal{H}}$, we complete the proof.

Lemma 3.5. Assume (H1)-(H4) hold. Then, the semigroup $\{S(t)\}_{t \geq 0}$ defined by (3.1) is asymptotically smooth in $\mathcal{H}$.

Proof. The process of the proof is the same as that of Lemma 4.3 in [5]. So, we omit the details here.

Our main result of this section reads as:

Theorem 3.6. Under the conditions (H1)-(H4), the semigroup $\{S(t)\}_{t \geq 0}$ corresponding to problem 2.4 2.8 has a global attractor in $\mathcal{H}$.

Proof. Lemmas 3.3, 3.5 and Theorem 2.3 guarantee the existence of a global attractor.

\section{Finite-dimensional attractor}

In this section we prove the finite dimensionality of the attractors given in Theorem 3.6 making use of Theorem 2.4

Lemma 4.1. Let the conditions (H1)-(H4) hold. If we assume subcritical conditions

$$
2 \leq p<\frac{2 N-2}{N-2} \text { if } N \geq 3 \text { and } 0<m<\frac{4}{N-4} \text { if } N \geq 5,
$$

then the dynamical system $(S(t), \mathcal{H})$ defined by (3.1) is quasi-stable on any bounded positively invariant set $B \subset \mathcal{H}$.

Proof. Theorem 2.1(i) ensures that the dynamical system $(S(t), \mathcal{H})$ satisfies 2.9 and (2.10) by considering $X=V_{2}, Y=V_{0}$, and $Z=L^{2}(\Omega \times(0,1))$. Furthermore, we observe from Theorem 2.1 (ii) that $(S(t), \mathcal{H})$ satisfies 2.11). Now, it remains to show that $(S(t), \mathcal{H})$ satisfies 2.12). Let $B_{0} \subset \mathcal{H}$ be a bounded set positively invariant with respect to $S(t)$. Let $S(t) y_{0}=\left(u, u_{t}, z\right)$ and $S(t) \widetilde{y_{0}}=\left(\widetilde{u}, \widetilde{u}_{t}, \widetilde{z}\right)$ for $y_{0} \in B_{0}$ and $\widetilde{y_{0}} \in B_{0}$, respectively. Define the seminorm

$$
n_{X}(u)=\|\nabla u\|_{2(p-1)}+\|u\|_{2(m+1)},
$$


then $n_{X}(\cdot)$ is a compact seminorm on $X$ because the embeddings $V_{2} \hookrightarrow W_{0}^{1,2(p-1)}(\Omega)$ and $V_{2} \hookrightarrow L^{2(m+1)}(\Omega)$ are compact. Hence, (3.11) can be rewritten as

$$
\left\|S(t) y_{0}-S(t) \widetilde{y_{0}}\right\|_{\mathcal{H}}^{2} \leq b(t)\left\|y_{0}-\widetilde{y_{0}}\right\|_{\mathcal{H}}^{2}+c(t) \sup _{0<s<t}\left(n_{X}(u(s)-\widetilde{u}(s))\right)^{2},
$$

where $b(t)=c e^{-\omega t}$ and $c(t)=C\left(B_{0}\right) \int_{0}^{t} e^{-\omega(t-s)} d s$. Moreover we see that $b \in L^{1}\left(\mathbb{R}^{+}\right)$, $\lim _{t \rightarrow \infty} b(t)=0$, and $c(t)$ is locally bounded on $[0, \infty)$ because $B_{0}$ is bounded.

Our desired result of this section is the following:

Theorem 4.2. Let the conditions of Lemma 4.1 hold. Then the global attractor $\mathcal{A}$ given in Theorem 3.6 has finite fractal dimension.

Proof. Since the global attractor $\mathcal{A}$ given in Theorem 3.6 is a bounded positively invariant set of $\mathcal{H}$, Lemma 4.1 yields that the dynamical system $(S(t), \mathcal{H})$ defined 3.1 is quasistable on $\mathcal{A}$. Thus, Theorem 2.4 implies that $\mathcal{A}$ has finite fractal dimension.

\section{Acknowledgments}

This work was supported by the National Research Foundation of Korea (NRF) grant funded by the Korea government (MSIP) (No. 2017R1A2B4004163).

\section{References}

[1] D. Andrade, M. A. Jorge Silva and T. F. Ma, Exponential stability for a plate equation with p-Laplacian and memory terms, Math. Methods Appl. Sci. 35 (2012), no. 4, 417426.

[2] I. Chueshov and I. Lasiecka, Von Karman Evolution Equations: Well-posedness and long-time dynamics, Springer Monographs in Mathematics, Springer, New York, 2010.

[3] R. Datko, Not all feedback stabilized hyperbolic systems are robust with respect to small time delays in their feedbacks, SIAM J. Control Optim. 26 (1988), no. 3, 697-713

[4] M. A. Jorge Silva and T. F. Ma, On a viscoelastic plate equation with history setting and perturbation of p-Laplacian type, IMA J. Appl. Math. 78 (2013), no. 6, 11301146.

[5] _ Long-time dynamics for a class of Kirchhoff models with memory, J. Math. Phys. 54 (2013), no. 2, 021505, 15 pp. 
[6] M. A. Jorge Silva, J. E. Muñoz Rivera and R. Racke, On a class of nonlinear viscoelastic Kirchhoff plates: well-posedness and general decay rates, Appl. Math. Optim. 73 (2016), no. 1, 165-194.

[7] M. Kirane and B. Said-Houari, Existence and asymptotic stability of a viscoelastic wave equation with a delay, Z. Angew. Math. Phys. 62 (2011), no. 6, 1065-1082.

[8] T. F. Ma and J. A. Soriano, On weak solutions for an evolution equation with exponential nonlinearities, Nonlinear Anal. 37 (1999), no. 8, Ser. A: Theory Methods, 1029-1038.

[9] M. I. Mustafa, Asymptotic behavior of second sound thermoelasticity with internal time-varying delay, Z. Angew. Math. Phys. 64 (2013), no. 4, 1353-1362.

[10] S. Nicaise and C. Pignotti, Stability and instability results of the wave equation with a delay term in the boundary or internal feedbacks, SIAM J. Control Optim. 45 (2006), no. $5,1561-1585$.

[11] _ Interior feedback stabilization of wave equations with time dependent delay, Electron. J. Differential equations 2011 (2011), no. 41, 20 pp.

[12] S. H. Park, Stability for a viscoelastic plate equation with p-Laplacian, Bull. Korean Math. Soc. 52 (2015), no. 3, 907-914.

[13] Energy decay for a von Karman equation with time-varying delay, Appl. Math. Lett. 55 (2016), 10-17.

[14] J. Y. Park, H. M. Kim and S. H. Park, On weak solutions for hyperbolic differential inclusion with discontinuous nonlinearities, Nonlinear Anal. 55 (2003), no. 1-2, 103113.

[15] Z. Yang, Existence and energy decay of solutions for the Euler-Bernoulli viscoelastic equation with a delay, Z. Angew. Math. Phys. 66 (2015), no. 3, 727-745.

[16] Y. Zhijian, Longtime behavior for a nonlinear wave equation arising in elasto-plastic flow, Math. Methods Appl. Sci. 32 (2009), no. 9, 1082-1104.

[17] Y. Zhijian and J. Baoxia, Global attractor for a class of Kirchhoff models, J. Math. Phys. 50 (2009), no. 3, 032701, 29 pp.

Sun-Hye Park

Office for Education Accreditation, Pusan National University, Busan 609-735, Korea E-mail address: sh-park@pusan.ac.kr 